\title{
Very Long-term Outcome after Linear vs. Electrogram-guided Ablation for Persistent Atrial Fibrillation: A Propensity Score-matched Analysis
}

Seigo Yamashita ( $\nabla$ seigoy722@yahoo.co.jp )

The Jikei University School of Medicine

Michifumi Tokuda

The Jikei University School of Medicine

Saagar Mahida

Liverpool Heart and Chest Hospital

Hidenori Sato

The Jikei University School of Medicine

Hirotsugu Ikewaki

The Jikei University School of Medicine

Hirotsuna Oseto

The Jikei University School of Medicine

Masaaki Yokoyama

The Jikei University School of Medicine

Ryota Isogai

The Jikei University School of Medicine

Kenichi Tokutake

The Jikei University School of Medicine

Kenichi Yokoyama

The Jikei University School of Medicine

Ryohsuke Narui

The Jikei University School of Medicine

Mika Kato

The Jikei University School of Medicine

Shin-ichi Tanigawa

The Jikei University School of Medicine

Ken-ichi Sugimoto

The Jikei University School of Medicine

Michihiro Yoshimura

The Jikei University School of Medicine

Teiichi Yamane 
The Jikei University School of Medicine

\section{Research Article}

Keywords: PsAF, LINE-group, pulmonary vein isolation (PVI), complex fractionated atrial electrogram [CFAE], EGM-guided

Posted Date: July 13th, 2021

DOl: https://doi.org/10.21203/rs.3.rs-692564/v1

License: (c) (1) This work is licensed under a Creative Commons Attribution 4.0 International License. Read Full License 


\section{Abstract}

The optimal ablation strategy for persistent atrial fibrillation (PSAF) remains to be defined. We sought to compare very long-term outcomes between linear ablation and electrogram (EGM)-guided ablation for PsAF. In a retrospective analysis, long-term arrhythmia-free survival compared between two propensityscore matched cohorts, one with pulmonary vein isolation (PVI) and linear ablation including roof/mitral isthmus line (LINE-group, $n=52$ ) and one with PVI and EGM-guided ablation (EGM-group; $n=52$ ). Overall, $99 \%$ of patients underwent successful PVI. Complete block following linear ablation was achieved for $94 \%$ of roof lines and $81 \%$ of mitral lines (both lines blocked in $75 \%$ ). AF termination by EGM-guided ablation was accomplished in $40 \%$ of patients. Non-PV foci were targeted in $7(13 \%)$ in the LINE-group and $5(10 \%)$ patients in the EGM-group $(p=0.76)$. During $100 \pm 28$ months of follow-up, linear ablation was associated with superior arrhythmia-free survival after the initial and last procedure $(1.8 \pm 0.9$ procedures) compared with EGM-group (Logrank test: $\mathrm{P}=0.0001$ and $\mathrm{P}=0.045$, respectively). In multivariable analysis, longer AF duration and EGM-guided ablation remained as independent predictors of AAs recurrence. Linear ablation might be a more effective complementary technique to PVI than EGMguided ablation for PsAF ablation.

\section{Introduction}

While pulmonary vein isolation $(\mathrm{PVI})$ is associated with high success rates in patients with paroxysmal atrial fibrillation (PAF), ${ }^{1-4}$ ablation outcomes in patients with persistent AF (PsAF) are more modest. In patients with PsAF, additional ablation strategies targeting AF substrate demonstrated the possible benefit in the previous meta-analysis of controlled studies. ${ }^{5}$ Linear ablation including roof and mitral isthmus (MI) lines ${ }^{6}$ and electrogram (EGM)-guided ablation 7,8 techniques have been widely used in this context. However, the reported outcomes following ablation with these techniques have been variable. ${ }^{9,10}$ In a previous multicenter randomized trial, additional AF substrate ablation (roof and MI lines or complex fractionated atrial electrogram [CFAE] or EGM-guided) beyond PVI for PsAF was not associated with incremental benefit compared to PVI alone. Importantly, while AF/atrial tachycardia (AT)-free survival rates were relatively low (around $40 \%$ ), operator bias for linear ablation and CFAE-guided ablation should be considered. ${ }^{11}$ There is a paucity of data on the relative efficacy of linear ablation and CFAE or EGMguided ablation for PsAF. ${ }^{11,12}$ In the present study we compared very long-term clinical outcomes of linear ablation and EGM-guided ablation.

\section{Results}

\section{Patient characteristics}

In our overall cohort of 104 patients the mean age was $53 \pm 11$ years old with only $2(1.9 \%)$ females. The average minimum continuous AF duration was $15 \pm 20$ months. 29 (28\%) had PsAF lasting between 6 months and one year while $43(41 \%)$ had PSAF for $>1$ year, Mean LAD and LVEF were $42 \pm 4.9 \mathrm{~mm}$ and 
$62 \pm 7.5 \%$, respectively. The number of failed AADs before CA was $1.4 \pm 1.0$ (including, 65 (78\%) on the class III AAD; Bepridil (150-200 mg) and 3 (2.9\%) on amiodarone). Beta-blocker was used in $39 \%$ of patients. Patient characteristics after propensity score-matched analysis (final cohort of 52 patients in the LINE-group and EGM-group) are shown in Table 1.

Table 1

Baseline patients' characteristics

\begin{tabular}{|c|c|c|c|c|}
\hline & $\begin{array}{l}\text { Total } \\
(n=104)\end{array}$ & $\begin{array}{l}\text { LINE-group } \\
(n=52)\end{array}$ & $\begin{array}{l}\text { EGM-group } \\
(n=52)\end{array}$ & p-value \\
\hline Age, years old & $53 \pm 11$ & $52 \pm 10$ & $54 \pm 12$ & 0.49 \\
\hline Female, n (\%) & $2(1.9 \%)$ & $2(4 \%)$ & $0(0 \%)$ & 0.50 \\
\hline Minimum continuous AF duration, month & $15 \pm 20$ & $15 \pm 18$ & $16 \pm 21$ & 0.80 \\
\hline Long-standing PsAF (> 1 year), $n$ & $43(41 \%)$ & $22(42 \%)$ & $21(40 \%)$ & 1.00 \\
\hline Unknown time of $\mathrm{AF}$ onset, $\mathrm{n}$ & $78(75 \%)$ & $42(81 \%)$ & $36(69 \%)$ & 0.26 \\
\hline $\mathrm{BMI}, \mathrm{kg} / \mathrm{m} 2$ & $25 \pm 3.7$ & $26 \pm 4.2$ & $25 \pm 3.3$ & 0.35 \\
\hline Number of failed AADs, $n$ & $1.4 \pm 1.0$ & $1.3 \pm 1.0$ & $1.5 \pm 1.0$ & 0.50 \\
\hline Structure heart disease, $\mathrm{n}$ & $8(8 \%)$ & $5(10 \%)$ & $3(6 \%)$ & 0.72 \\
\hline CHADS2 score, $n$ & $0.7 \pm 0.8$ & $0.7 \pm 0.7$ & $0.7 \pm 0.8$ & 1.00 \\
\hline Hypertension, $\mathrm{n}$ & $53(51 \%)$ & $27(52 \%)$ & $26(50 \%)$ & 1.00 \\
\hline Diabetes Mellitus, $\mathrm{n}$ & $10(10 \%)$ & $5(10 \%)$ & $5(10 \%)$ & 1.00 \\
\hline LAD, mm & $42 \pm 4.9$ & $42 \pm 5.1$ & $41 \pm 4.7$ & 0.31 \\
\hline LVEF, \% & $62 \pm 7.5$ & $62 \pm 8.2$ & $63 \pm 7.0$ & 0.55 \\
\hline BNP, pg/ml & $85 \pm 61$ & $82 \pm 57$ & $91 \pm 65$ & 0.49 \\
\hline Ccr, mg/dl & $80 \pm 17$ & $81 \pm 17$ & $78 \pm 18$ & 0.41 \\
\hline
\end{tabular}

\section{Acute procedural outcomes}

As shown Table 2, 12 (23\%) patients in LINE-group and 5 (10\%) patients in EGM-group were in SR at the beginning of the procedure. In the EGM-group, AF was induced by burst pacing in all patients with SR at the beginning of the procedure. PVI was performed as a first step in all patients, with a $99 \%$ of successful isolation rate. In the LINE-group electrical cardioversion was performed after PVI in all patients. In the EGM-group AF persisted post-PVI in all but 1 patient. 
Table 2

Ablation results in the initial CA.

\begin{tabular}{|llll|}
\hline & $\begin{array}{l}\text { LINE-group } \\
(\mathbf{n = 5 2 )}\end{array}$ & $\begin{array}{l}\text { EGM-group } \\
(\mathbf{n = 5 2 )}\end{array}$ & p-value \\
\hline Start in AF rhythm, $\mathrm{n}$ & $40(77 \%)$ & $47(90 \%)$ & 0.11 \\
\hline Successful PVI (No. of PVs) & $204 / 206(99 \%)$ & $203 / 205(99 \%)$ & 1.00 \\
\hline Successful roof line, $\mathrm{n}$ & $49 / 52(94 \%)$ & $2 / 3(67 \%)$ & - \\
\hline Successful MI line, $\mathrm{n}$ & $42 / 52(81 \%)$ & $4 / 4(100 \%)$ & - \\
\hline AF termination by EGM-guide ablation, $\mathrm{n}$ & - & $21(40 \%)$ & - \\
\hline SVC isolation, $\mathrm{n}$ & $2(4 \%)$ & $1(2 \%)$ & 1.00 \\
\hline Non-PV foci except for SVC, $\mathrm{n}$ & $6(12 \%)$ & $4(8 \%)$ & 0.74 \\
\hline Procedure time, min & $252 \pm 65$ & $291 \pm 56$ & 0.002 \\
\hline Fluoroscopic time, min & $137 \pm 41$ & $141 \pm 32$ & 0.64 \\
\hline Total RF time, min & $65 \pm 27$ & $91 \pm 34$ & $<0.001$ \\
\hline RF time for PVI, min & $32 \pm 25$ & $36 \pm 15$ & 0.38 \\
\hline Major complications, $\mathrm{n}$ & $3(5.8 \%)$ & $2(3.8 \%)$ & 1.00 \\
\hline Very early recurrence of AF (<3d), $\mathrm{n}$ & $16(31 \%)$ & $23(44 \%)$ & 0.22 \\
\hline $\begin{array}{l}\text { Abbreviations; AF, atrial fibrillation; Ml, mitral isthmus; PVI, pulmonary } \\
\text { radiofrequency: SVC, superior vena cava. }\end{array}$ & & & \\
\hline
\end{tabular}

In LINE-group, roof and MI lines were successfully blocked in 49 (94\%) and 42 (81\%) patients, respectively. Both lines were completed in 39 (75\%) patients. Epicardial ablation within the CS for the MI line was added in 28 (54\%) patients. Mean RF time for roof and Ml line was $6.6 \pm 3.5$ and $14.0 \pm 7.3$ minutes, respectively. In EGM-group, AF was terminated by RF in $21(40 \%)$ patients (conversion to AT in 11 patients and termination to SR in 10 patients). Mean RF time for EGM-guide ablation was $47 \pm 30$ minutes. The right atrium was targeted in $36(69 \%)$ patients. Roof and/or MI lines were created due to induced roof and/or Ml line-dependent ATs in 5 patients.

Non-PV foci ablation, including SVC isolation, was performed in 7 (13\%) in the LINE-group and 5 (10\%) patients in the EGM-group $(p=0.76)$. Procedure time and total RF time was significantly longer in EGMgroup (LINE-group vs. ECG-group; procedure time $252 \pm 65$ vs. $291 \pm 56$ minutes, $p=0.002$; RF time $65 \pm$ 27 vs. $91 \pm 34$ minutes, $p<0.001)$. There were no differences in the incidence of procedure-related major complications between the two groups. Very early recurrence rates (within 3 days) were also comparable between two groups (Table 2).

\section{Long-term outcome}


During $100 \pm 28$ months of follow-up, the LINE-group had superior arrhythmia-free survival after the index ablation compared to the EGM-group (Logrank test: $P=0.0001$, Fig. $1 \mathrm{~A}$ ). SR maintenance was consistently higher in the LINE-group compared to the EGM-group (LINE-group vs. ECG-group; year 1: 79\% vs. $42 \%$; year 3: $63 \%$ vs. $31 \%$; year 5 : $60 \%$ vs. $25 \%$; year 8 : $54 \%$ vs. $19 \%$ ). AA recurrence subtypes were similar between the LINE-group vs. EGM-groups (LINE-group vs. ECG-group; AF-only recurrence: 11 [46\%] vs. 28 [66\%]; AT-only: 5 [21\%] vs. 7 [17\%]; AF and AT 8 [33\%] vs. 7 [17\%], p = 0.21 Fig. 2). In addition, the pattern of AF recurrence was comparable between the two groups (LINE-group vs. ECG-group; PsAF recurrence 8 [42\%] vs. $15[44 \%], p=1.00)$.

Among $24(46 \%)$ and $42(81 \%)$ patients of AAs recurrence in LINE and EGM-group, 24 (100\%) and 34 $(81 \%)$ of patients underwent the re-do procedures with $1.6 \pm 0.7$ and $2.0 \pm 1.0$ procedures $(p=0.02)$. In LINE-group, mean number of reconnected PVs were $2.8 \pm 0.9 \mathrm{PVs} /$ patient, and reconnection of roof and MI lines was observed in $9(38 \%)$ and 13 (54\%) patients during the second procedure. All reconnected PVs were successfully re-isolated and bidirectional block was established across all lines. EGM-guided ablation was added in 7 (29\%) patients. In EGM-group, all reconnected PVs (2.9 $\pm 0.9 \mathrm{PVs} /$ patient) were also successfully re-isolated, and additional roof and MI lines were created in 24 (71\%) and $27(79 \%)$ patients during the second procedure. Non-PV foci, (including SVC in a subset) were detected in 21 (36\%) patients. The incidence of non-PV triggers was comparable between the two groups (LINE-group vs. EGMgroup: 8 [36\%] vs. 13 [38\%], p = 0.92). However, multiple ATs were more frequently induced during the second procedure in EGM-group (LINE-group vs. EGM-group: 7 [21\%] vs. 0 [0\%], p = 0.03). After taking multiple procedures into account (1.8 \pm 0.9 procedures), $66 / 104(63 \%)$ maintained SR in this population. AAs-free survival was remained significantly higher in LINE-group as compared to EGM-group after the last procedure (LINE-group vs. EGM-group: year 1: $96 \%$ vs. $88 \%$; year 3: $92 \%$ vs. $71 \%$; year 5 : $83 \%$ vs. $62 \%$; year 8: logrank test: $88 \%$ vs. $65 \%, p=0.045$, Fig. $1 B$ ). In addition, fewer CA procedures were required to maintain SR in the LINE-group as compared to EGM-group (1.6 \pm 0.7 vs. $2.0 \pm 1.0, p=0.02$ ).

\section{Predictors of AAs recurrence after the initial CA}

In univariable analysis, longer AF duration, EGM-guide ablation and AF at the beginning of the index CA procedure were identified as predictors of AAs recurrence after the index ablation. Of note, incomplete line block and non-termination during EGM-guide ablation did predict AAs recurrence. In multivariable analysis, longer AF duration and EGM-guided ablation remained as independent predictors of AAs recurrence (OR: $1.01,95 \% \mathrm{Cl}: 1.00-1.03, p=0.015$, OR: $2.45,95 \% \mathrm{Cl}: 1.47-4.09, \mathrm{p}=0.0007$, respectively, Table 3). 
Table 3

Predictors of AAs recurrence after the initial CA

\begin{tabular}{|c|c|c|c|c|c|c|}
\hline & \multicolumn{3}{|c|}{ Univariable analysis } & \multicolumn{3}{|c|}{ Multivariable analysis } \\
\hline & OR & $95 \% \mathrm{Cl}$ & $\begin{array}{l}\mathrm{P}- \\
\text { value }\end{array}$ & OR & $95 \% \mathrm{Cl}$ & $\begin{array}{l}\mathrm{P}- \\
\text { value }\end{array}$ \\
\hline Age, years old & 0.71 & $\begin{array}{l}0.97- \\
1.02\end{array}$ & 0.71 & & & \\
\hline $\begin{array}{l}\text { Minimum continuous AF duration, } \\
\text { month }\end{array}$ & 1.01 & $1.00-1.03$ & 0.033 & 1.01 & $1.00-1.03$ & 0.015 \\
\hline Unknown time of $\mathrm{AF}$ onset & 0.96 & $\begin{array}{l}0.55- \\
1.66\end{array}$ & 0.87 & & & \\
\hline $\mathrm{BMI}, \mathrm{kg} / \mathrm{m} 2$ & 0.99 & $\begin{array}{l}0.93- \\
1.06\end{array}$ & 0.82 & & & \\
\hline Hypertension & 0.96 & $\begin{array}{l}0.60- \\
1.56\end{array}$ & 0.88 & & & \\
\hline CHADS2 score & 0.27 & $\begin{array}{l}0.61- \\
1.15\end{array}$ & 0.26 & & & \\
\hline LAD, mm & 1.01 & $\begin{array}{l}0.96- \\
1.06\end{array}$ & 0.82 & & & \\
\hline LVEF, \% & 1.00 & $\begin{array}{l}0.97- \\
1.03\end{array}$ & 0.95 & & & \\
\hline BNP, pg/ml & 1.00 & $0.99-1.00$ & 0.95 & & & \\
\hline Ccr, $\mathrm{ml} / \mathrm{min}$ & 0.99 & $0.98-1.00$ & 0.16 & & & \\
\hline Start in AF rhythm & 2.24 & $\begin{array}{l}1.03- \\
4.90\end{array}$ & 0.044 & 1.78 & $\begin{array}{l}0.80- \\
3.94\end{array}$ & 0.16 \\
\hline EGM-guided ablation & 2.62 & $\begin{array}{l}1.59- \\
4.34\end{array}$ & 0.0002 & 2.45 & $\begin{array}{l}1.47- \\
4.09\end{array}$ & 0.0007 \\
\hline Non-PV foci including SVC & 1.46 & $\begin{array}{l}0.73- \\
2.95\end{array}$ & 0.29 & & & \\
\hline
\end{tabular}

\section{Discussion}

The present study is the first report to directly compare the very long-term clinical outcome, spanning 8years, between linear ablation (roof and MI lines) and EGM-guide ablation in addition to PVI for PsAF. Using a propensity score-matched analysis, we demonstrated superiority of linear ablation to EGM-guided ablation following single and multiple procedures. In addition, in addition to continuous AF duration, 
EGM-guided ablation was an independent predictor of arrhythmia recurrence after the index ablation procedure.

\section{Linear ablation for AF}

Multiple previous studies have reported that linear ablation for PSAF is associated with favorable medium term outcomes, with success rates of $69-88 \%$ at 1-1.5 year after CA. ${ }^{6,18,19}$ Gaita et al. reported that PVI plus linear ablation is superior to $\mathrm{PVI}$ alone in maintaining SR during a maximum follow-up period of 3 years. ${ }^{20}$ while the superiority of linear ablation have not been consistently demonstrated compared with $\mathrm{PVI}$ alone. ${ }^{9,10,21}$ The concept of linear ablation was derived from the evidence of high success rate of the surgical MAZE procedure. ${ }^{22}$ Roof and MI lines have a potential benefit for alteration of AF wavelet propagation and elimination of spectral components by complete bidirectional block of linear lesions. In addition, these linear lesions lead to reduction of excitable LA myocardial mass, the attenuation of vagal innervation ${ }^{23}$ and elimination of non-PV foci, especially relating to the ligament of Marshall. ${ }^{24,25}$ Although the endpoint of linear ablation is clear, establishing linear block, especially during MI line ablation, maybe challenging. Incomplete linear ablation has been associated with the occurrence of macro-reentrant $\mathrm{AT}^{26,27}$ and $\mathrm{AF}$ recurrences. ${ }^{28,29}$ In the present study, although acute success rates of linear ablation were relatively high ( $94 \%$ in roof line, $81 \%$ in $\mathrm{MI}$ line), roof and $\mathrm{MI}$ lines were reconnected in $38 \%$ and $54 \%$ of patients at re-do procedures. Nevertheless, the incidence of linear gap-related AT was observed in only $13 \%$, which was statistically similar with EGM-guided ablation group. These results may suggest that complete linear block is important for the modification of AF substrate, rather than for avoiding occurrence of macro-reentrant AT. It was possible to create durable linear lesions by using steerable sheaths in our study compared with other previous studies, which may have led to a better clinical outcomes.

\section{EGM (CFAE)-guided ablation for AF}

The original CFAE ablation study, reported by Nademanee et al., demonstrated high acute AF termination rates of $82 \%$ and arrhythmia-free survival in three quarters of patients at one-year follow-up. ${ }^{7}$ Haissaguerre et al. subsequently proposed EGM-guided ablation targeting fractionated, rapid and gradient signals in addition to linear ablation, an approach associated with a high acute AF termination rate. ${ }^{8}$ The concept of EGM or CFAE-guided ablation involves targeting AF driver sources represented by pivot points of wavelets, continuous re-entry of fibrillation waves with relatively short cycle lengths, and heterogenous temporal and special distribution. ${ }^{30}$ Of note however, identification of CFAE signals is quite subjective (e.g. definition of fractionated electrograms) and therefore this strategy was not consistent and not formally established, even with the use of automatic analysis algorithms incorporated into 3DEAM systems. ${ }^{21,31} \mathrm{~A}$ recent large meta-analysis did not demonstrate an incremental beneficial effect of CFAE ablation in addition to PVI compared with PVI alone in PSAF patients. ${ }^{9}$ It is possible that in addition to critical AF drivers CFAEs represent also passive activations, such as signal artifact, double potential, slow conduction and wavebreak. ${ }^{15,32}$ In the present study, EGM-guided ablation, using the Bordeaux 
approach, ${ }^{8}$ was performed by visual and manual inspection by sufficiently experienced operator. However, methodological differences might be associated with lower AF termination rate (40\%) and poor clinical outcome (AF/AT-free survival of 38\% /1.5 years). An important point to consider when comparing outcomes of CFAE ablation is the inter-operator variability CFAE identification.

\section{Direct comparison of clinical outcome between linear and EGM (CFAE)-guided ablation}

Although previous meta-analyses comparing different strategies for PsAF ablation in the different cohorts reported non-superiority of additional substrate modification beyond PVI, the studies were associated with heterogenous patient populations and follow-up periods. ${ }^{9,21}$ Only two previous studies have prospectively compared linear ablation and CFAE ablation in PSAF patients. In contrast to our results, both studies reported no significant differences in arrhythmia-free survival between the two groups during medium-term follow-up. ${ }^{11,12}$ While Estner et al. reported similar outcomes between linear ablation and CFAE ablation at 1 year, the success rates were lower than those in the present study (linear vs. CFAE: $37 \%$ vs. $39 \%)$ despite high success rates of linear block $(100 \%)$ and AF termination ( $82 \%)$ during CFAE ablation. Of note, their ablation strategy in the linear ablation group included roof and anterior lines in only two thirds of patients, and mean LA size was larger (47-49 mm). ${ }^{12}$ These results may suggest that the effect of linear ablation, especially anterior line ablation, is limited in advanced PSAF. In the STAR-AFII trial, there was no difference in AF/AT-free survival between linear ablation (roof and $\mathrm{MI}$ lines) and CFAE ablation during 1.5 years of follow-up ( $41 \%$ vs. $37 \%$ ). Regarding procedural results, AF terminated in $45 \%$ by CFAE ablation, and successful linear ablation was achieved in $93 \%$ and $75 \%$ of roof and $\mathrm{Ml}$ lines, respectively. ${ }^{11}$ There are a number of potential explanations for the inconsistent results between our study and STAR AF II. Firstly, the acute success rate of MI line block was relatively lower than our study (81\%). Secondly, our ablation protocol included ablation for non-PV foci. Thirdly, we confirmed elimination of dormant conduction post-PVI with isoproterenol and adenosine after 30 minutes waiting in all patients. Fourthly, a steerable sheath was used for linear ablation, which may have contributed to the higher successful rate in the linear ablation group. ${ }^{33}$ Overall, our study showed consistent efficacy of linear ablation in PsAF patients during very long-term follow-up (> 8 years) after the initial and multiple catheter ablation procedures with fewer procedures compared to the EGM-guided ablation cohort. Multiple ATs was more frequently induced at the re-do procedure in the EGM-guided ablation group. These findings may suggest that some of the critical CFAE areas were not targeted during the index ablation, ${ }^{15,32}$ and potential incompletely ablated areas may create a substrate for iatrogenic arrhythmias. ${ }^{34}$

We have several limitations in the present study. First, the study has the inherent limitations of a nonrandomized retrospective study from a single-center. However, we used propensity score-matched analysis to compare different ablation methods, therefore patients' backgrounds were statistically adjusted. Second, we used an irrigated ablation catheter without contact force in this study. Contact force 
guide ablation makes it possible to more firmly create transmural lesion for PVI and linear ablation compared with conventional ablation catheter, which may affect the results.

\section{Conclusions}

Our single center retrospective analysis demonstrated that linear ablation might be a more effective complementary technique to PVI than EGM-guided ablation with more favorable outcomes during very long-term follow-up period.

\section{Methods}

\section{Study population}

A total of 325 PsAF patients who underwent a first-time catheter ablation (CA) in our institution between September 2008 and July 2015 were retrospectively included. In this population, PVI plus linear ablation or EGM-guided ablation was performed in 230 and 82 patients, respectively. Following propensitymatched score analysis, 104 patients (LINE-group: 52, EGM-group: 52) were included in the analysis. PsAF was defined as AF lasting more than 7 days with or without anti-arrhythmic drugs, and longstanding persistent $A F$ was defined as continuous $A F$ for more than 12 months. ${ }^{13}$ AF duration was defined as the duration from the date when the AF was detected on 12-lead electrocardiogram (ECG) to either the date of AF termination by medical/electrical cardioversion, or the date of the initial CA procedure (if AF persisted despite medical/electrical cardioversion).

Clinical investigations were conducted in accordance with the principles expressed in the Declaration of Helsinki. All data were compliant with the International Conference on Harmonization guidelines. The study was approved by the ethics committee of The Jikei University School of Medicine for Biomedical Research. All methods were carried out in accordance with relevant guideline and regulations. All patients provided their written informed consent.

\section{Catheter ablation (CA)}

Pre-procedure preparation and techniques for PsAF ablation have been described previously. ${ }^{14,15}$ In brief, all patients received oral anticoagulation at least 4 weeks prior to the ablation procedure. Anti-arrhythmic drugs were discontinued for at least five half-lives prior to ablation. After exclusion of left atrial (LA) thrombus using trans-esophageal echocardiography, CA was performed under mild deep sedation. Continuous and bolus heparin administered with a target activated clotting time of 300-400 seconds.

The initial ablation step involved segmental antral PVI (in AF or SR) with the guidance of a large circular mapping catheter (Lasso 20-30 mm; Biosense-Webster Inc, Diamond Bar, CA, USA). Ablation was performed using a non-contact force sensing open-irrigated $3.5 \mathrm{~mm}$-tip ablation catheter (Cool Path ${ }^{\text {TM }}$ Duo, FlexAbility ${ }^{\text {Tw}}$; St jude Medical or ThermoCool/SF; Biosense Webster) with a power limit of 25-35W. 
The procedural endpoint of PVI was bidirectional electrical conduction block between the LA and PVs. ${ }^{14}$ Following PVI, linear ablation or EGM-guided ablation was performed.

In LINE-group, following roof line ablation (connecting the superior PVs), MI line ablation was performed with the endpoint of a bidirectional electrical conduction block across the lines. ${ }^{16,17} \mathrm{MI}$ line ablation was performed from the mitral annulus to the lower pole of the left inferior PV along the shortest line located (3-4 o'clock in the left-anterior oblique view). Epicardial ablation within the coronary sinus (CS) was subsequently added in cases with incomplete block from endocardial ablation. Ablation setting during linear ablation was as follows: a power output of 30-40 W on the roof and endocardial MI and 20-25W on the epicardium within the CS.

In the EGM-group, ablation was performed at all sites displaying any of the following EGM features: 1) continuous electrical activity, 2) rapid activity with cycle length (CL) shorter than the mean LA appendage AF CL, 3) complex and fractionated electrograms, and 4) a gradient of activation (a temporal gradient of at least $70 \mathrm{~ms}$ between the distal and proximal bipoles on the map electrode, potentially representing a local circuit) with the endpoint of AF termination. ${ }^{8}$ The power output during EGM-guided ablation was $30 \mathrm{~W}$ except for the posterior wall adjacent to the esophagus (20W with ablation termination upon abolition of the local specific electrograms). Electrical cardioversion was performed at the end of the procedure where elimination of targeting signals in both right and left atrium failed to terminate AF.

In all cases, the presence/absence of dormant conduction was tested by administration of isoproterenol $(4 \mathrm{ug})$ and adenosine triphosphate $(10-20 \mathrm{mg})$ in each PV after a minimum waiting period of 30 minutes after PVI. Dormant conduction was eliminated by additional radiofrequency (RF) applications. In cases with evidence of non-PV foci, the ablation strategy involved targeting non-PV foci. Superior vena cava (SVC) isolation was performed if arrhythmogenicity was detected in the SVC. Sustained AT occurring during the procedure was also mapped and ablated. Carvotricuspid isthmus linear ablation was routinely performed in all patients regardless of the presence or absence of documented typical atrial flutter. 3Delectroanatomical mapping (EAM) systems (CARTO3, Biosense-Webster Inc. or Navx, St Jude Medical, Inc, Minneapolis, MN, USA) were used during CA.

In the case of re-do procedures, the ablation strategy involved re-isolation of pulmonary veins (where indicated), and/or additional EGM-guide ablation, and/or linear ablation (roof and MI lines where necessary) with operator's discretion. Non-PV foci were targeted during the re-do procedure in the same way as the index procedure (following administration of isoproterenol [4 ug] and adenosine triphosphate [10-20 mg]). In case with AT recurrence, the arrhythmia was targeted with AT non-inducibility as the endpoint (no AT inducible with burst pacing up to $200 \mathrm{~ms}$ ).

\section{Acute outcomes}

Acute procedure related complications (cardiac tamponade, air embolism and major bleeding) were documented. Very early recurrences were defined as recurrences occurring within three days of the procedure. 


\section{Follow-up}

Patients were followed-up with 12-lead ECG and 24-hour Holter ECG at 1, 3, 6 and 12 months and subsequently every 6-12 months. Amongst symptomatic patients in whom 24 -hour monitors failed to document arrhythmia, a 5-day event recorder or portable ECG recorder (HeartScan, Omron Healthcare, Kyoto, Japan) was used. The primary end point was a recurrence of atrial arrhythmias (AAs), which included AF or AT. AA recurrence was defined as the presence of AF/AT lasting $>30$ seconds after the blanking period of 3 months.

\section{Statistical analyses}

Due to differences in baseline characteristics between patients in the linear ablation versus EGM-guided ablation groups, propensity-score matching was used to identify a subsets of patients with similar baseline characteristics. Patients were matched in a 1:1 ratio based propensity scores. Propensity scores were calculated for each patient using multivariable logistic regression with following 14 covariates: age, gender, body mass index, $A F$ duration, long-standing $A F$, asymptomatic $A F$, the number of failed AADs, structure heart disease, brain natriuretic peptide (BNP), estimated glomerular filtration rate, hypertension, diabetes mellitus, congestive heart failure, CHADS2 score, LA diameter (LAD) and left ventricular ejection fraction (LVEF), using a caliper width equal to 0.2 of the standard deviation of the logit of the propensity score. Quantitative data were expressed as the mean \pm standard deviation. Comparisons between groups were performed using an unpaired Student's $t$-test or Wilcoxon's rank-sum test (based on the distribution of the values). Comparisons of means from the same individual were performed using a paired Student's $t$-test. Categorical data were compared by $\chi^{2}$ test. Kaplan-Meier curves were used to analyze freedom from AAs, and groups were compared using the log-rank test. The multivariable analysis was described by Cox regression for the assessment of the predictors of AAs recurrence after the initial CA procedure. All tests were 2-tailed, and $P$ values of $<0.05$ were considered to indicate statistical significance. All of the statistical analyses were performed using the MedCalc software package, version 11.2 (MedCalc Software, Mariakerke, Belgium).

\section{Declarations}

\section{AUTHOR CONTRIBUTION}

S.Y. and T.Y. wrote the main manuscript, and the other authors worked for collection of study data. All authors reviewed and approved the final manuscript.

\section{CONFLICT OF INTEREST}

SY: None, MT: None, MS: None, HS: None, HI: None, HO: None, MY: None, RI: None, KT: None, KY: None, RN: None, MK: None, ST: None, KS: None, MY: There is no COI to disclose directly related to this study. Outside this study, Michihiro Yoshimura reports personal fees from Mochida Pharmaceutical Co., Ltd., personal fees from Daiichi Sankyo Co., Ltd., personal fees from Pfizer Japan Inc., personal fees from 
Kowa Co., Ltd., grants and personal fees from Mitsubishi Tanabe Pharma Corporation, grants form Teijin Pharma Ltd., grants from Astellas Pharma Inc., grants from Shionogi \& Co., Ltd., TY: There is no COI to disclose directly related to this manuscript. Speaker honoraria from DAIICHI SANKYO COMPANY, Ltd., Japan, Boerringer Ingelheim, Abbott Japan, Bristol-Myers Squibb, Medtronic Japan, and Japan LifeLine, and research grants from Boehringer Ingelheim.

\section{References}

1. Kuck, K. H. et al. FIRE AND ICE Investigators. Cryoballoon or Radiofrequency Ablation for Paroxysmal Atrial Fibrillation. N Engl J Med, 374, 2235-2245 (2016).

2. Fürnkranz, A. et al. Improved 1-year clinical success rate of pulmonary vein isolation with the secondgeneration cryoballoon in patients with paroxysmal atrial fibrillation. J Cardiovasc Electrophysiol, 25, 840-844 (2014).

3. Steinberg, J. S. et al. Very long-term outcome after initially successful catheter ablation of atrial fibrillation. Heart Rhythm, 11, 771-776 (2014).

4. Bhargava, M. et al. Impact of type of atrial fibrillation and repeat catheter ablation on long-term freedom from atrial fibrillation: results from a multicenter study. Heart Rhythm, 6, 1403-1412 (2009).

5. Baykaner, T. et al. Clinical Implications of Ablation of Drivers for Atrial Fibrillation: A Systematic Review and Meta-Analysis. Circ Arrhythm Electrophysiol, 11, e006119 https://doi.org/10.1161/CIRCEP.117.006119 (2018 May).

6. Willems, S. et al. Substrate modification combined with pulmonary vein isolation improves outcome of catheter ablation in patients with persistent atrial fibrillation: a prospective randomized comparison. Eur Heart J, 27, 2871-2878 (2006).

7. Nademanee, K. et al. A new approach for catheter ablation of atrial fibrillation: mapping of the electrophysiologic substrate. J Am Coll Cardiol, 43, 2044-2053 (2004).

8. Haïssaguerre, M. et al. Catheter ablation of long-lasting persistent atrial fibrillation: critical structures for termination. J Cardiovasc Electrophysiol, 16, 1125-1137 (2005).

9. Providência, R. et al. Is There Still a Role for Complex Fractionated Atrial Electrogram Ablation in Addition to Pulmonary Vein Isolation in Patients With Paroxysmal and Persistent Atrial Fibrillation? Meta-Analysis of 1415 Patients. Circ Arrhythm Electrophysiol, 8, 1017-1029 (2015).

10. Clarnette, J. A. et al. Outcomes of persistent and long-standing persistent atrial fibrillation ablation: a systematic review and meta-analysis. Europace, 20, f366-f376 (2018).

11. Verma, A. et al. STAR AF II Investigators. Approaches to catheter ablation for persistent atrial fibrillation. N Engl J Med, 372, 1812-1822 (2015).

12. Estner, H. L. et al. Complex fractionated atrial electrogram or linear ablation in patients with persistent atrial fibrillation-a prospective randomized study. Pacing Clin Electrophysiol, 34, 939-948 (2011). 
13. January, C. T. et al. 2014 AHA/ACC/HRS guideline for the management of patients with atrial fibrillation: a report of the American College of Cardiology/American Heart Association Task Force on practice guidelines and the Heart Rhythm Society., 130, e199-e267 (2014).

14. Yamane, T. et al. Segmental pulmonary vein antrum isolation using the "large-size" lasso catheter in patients with atrial fibrillation. Circ J, 71, 753-760 (2007).

15. Matsuo, S. et al. Substrate modification by pulmonary vein isolation and left atrial linear ablation in patients with persistent atrial fibrillation: its impact on complex-fractionated atrial electrograms. $J$ Cardiovasc Electrophysiol, 23, 962-970 (2012).

16. Hocini, M. et al. Techniques, evaluation, and consequences of linear block at the left atrial roof in paroxysmal atrial fibrillation. A prospective randomized study., 112, 3688-3696 (2005).

17. Jaïs, P. et al. Technique and results of linear ablation at the mitral isthmus., 110, 2996-3002 (2004).

18. Yao, Y. et al. Stepwise linear approach to catheter ablation of atrial fibrillation. Heart Rhythm, 4, 1497-1504 (2007).

19. Fassini, G. et al. Left mitral isthmus ablation associated with PV Isolation: long-term results of a prospective randomized study. J Cardiovasc Electrophysiol, 16, 1150-1156 (2005).

20. Gaita, F. et al. Long-term clinical results of 2 different ablation strategies in patients with paroxysmal and persistent atrial fibrillation. Circ Arrhythm Electrophysiol, 1, 269-275 (2008).

21. Scott, P. A., Silberbauer, J. \& Murgatroyd, F. D. The impact of adjunctive complex fractionated atrial electrogram ablation and linear lesions on outcomes in persistent atrial fibrillation: a meta-analysis. Europace, 18, 359-367 (2016).

22. Cox, J. L. et al. Current status of the MAZE procedure for the treatment of atrial fibrillation. Semin Thorac Cardiovasc Surg, 12, 15-19 (2000).

23. Kim, M. Y. et al. The ectopy-triggering ganglionated plexuses in atrial fibrillation. Auton Neurosci, 228, 102699 (2020 Nov).

24. Jais, P., Hsu, L. F., Hocini, M., Sanders, P. \& Haissaguerre, M. The left atrial isthmus: from dissection bench to ablation lab. J Cardiovasc Electrophysiol, 15, 813-814 (2004).

25. Derval, N. et al. Marshall bundle elimination, Pulmonary vein isolation, and Line completion for ANatomical ablation of persistent atrial fibrillation (Marshall-PLAN): Prospective, single-center study.Heart Rhythm. 2020 Dec29:S1547-5271(20)31218-2. doi: 10.1016/j.hrthm.2020.12.023. Online

26. Sawhney, N., Anousheh, R., Chen, W. \& Feld, G. K. Circumferential pulmonary vein ablation with additional linear ablation results in an increased incidence of left atrial flutter compared with segmental pulmonary vein isolation as an initial approach to ablation of paroxysmal atrial fibrillation. Circ Arrhythm Electrophysiol, 3, 243-248 (2010).

27. Matsuo, S. et al. Clinical predictors of termination and clinical outcome of catheter ablation for persistent atrial fibrillation. J Am Coll Cardiol, 54, 788-795 (2009).

28. Gaita, F. et al. Linear cryoablation of the left atrium vs. pulmonary vein cryoisolation in patients with permanent atrial fibrillation and valvular heart disease: correlation of electroanatomic mapping and 
long-term clinical results., 111, 136-142 (2005).

29. Gaita, F. et al. Very long-term results of surgical and transcatheter ablation of long-standing persistent atrial fibrillation. Ann Thorac Surg, 96, 1273-1278 (2013).

30. Konings, K. T. et al. High-density mapping of electrically induced atrial fibrillation in humans., $\mathbf{8 9}$, 1665-1680 (1994).

31. Scherr, D. et al. Automated detection and characterization of complex fractionated atrial electrograms in human left atrium during atrial fibrillation. Heart Rhythm, 4, 1013-1020 (2007).

32. de Bakker, J. M. T. \& Wittkampf, F. H. M. The pathophysiologic basis of fractionated and complex electrograms and the impact of recording techniques on their detection and interpretation. Circ Arrhythm Electrophysiol, 3, 204-213 (2010).

33. Matsuo, S. et al. Completion of mitral isthmus ablation using a steerable sheath: prospective randomized comparison with a nonsteerable sheath. J Cardiovasc Electrophysiol, 22, 1331-1338 (2011).

34. Shah, A. J. et al. Atrial tachycardias arising from ablation of atrial fibrillation: a proarrhythmic bump or an antiarrhythmic turn? Cardiol Res Pract, 2010, 950763 https://doi.org/10.4061/2010/950763 (2010).

\section{Figures}

(A) Lon-term clinical outcome after the initial CA

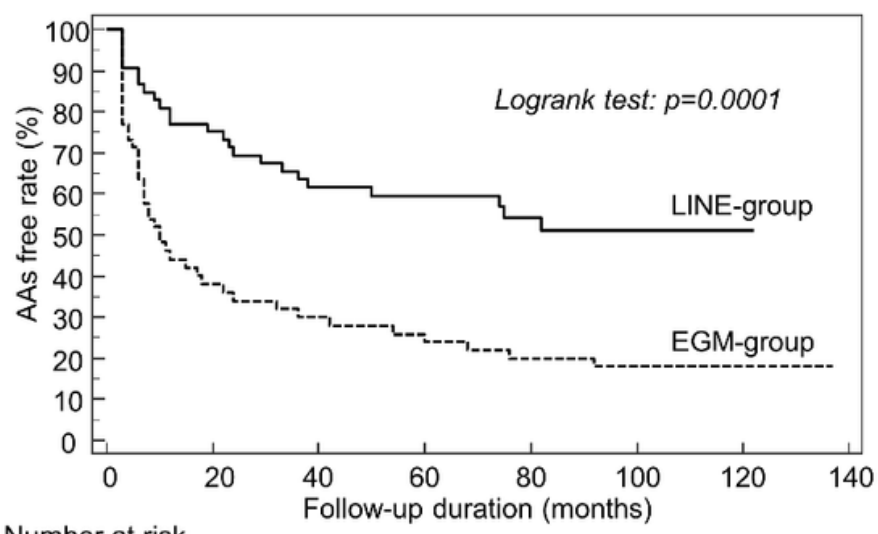

Number at risk

$\begin{array}{llllllll}\text { LINE } 52 & 39 & 32 & 25 & 18 & 10 & 2 & 0 \\ \text { EGM } 52 & 19 & 15 & 12 & 10 & 9 & 5 & 0\end{array}$

(B) Long-term clinical outcome after the last CA

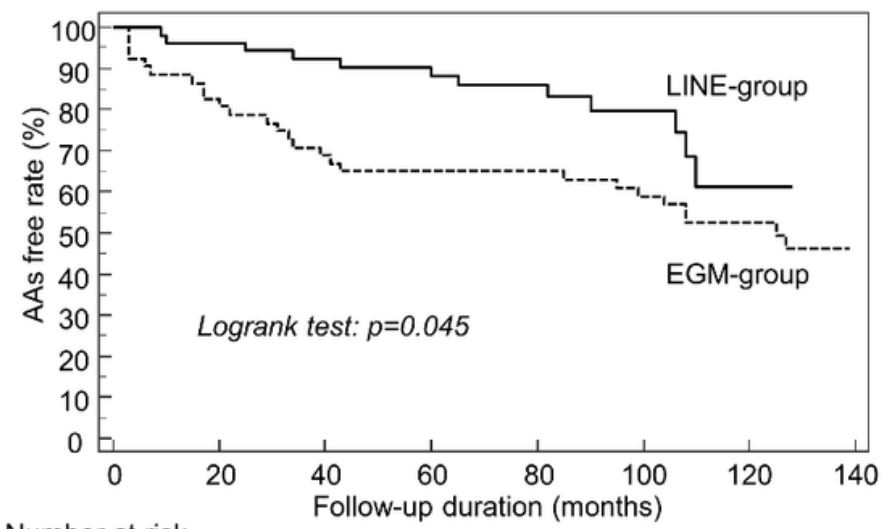

Number at risk

$\begin{array}{llllllll}\text { LINE } 52 & 50 & 48 & 42 & 32 & 18 & 5 & 0 \\ \text { EGM } 52 & 41 & 35 & 33 & 33 & 29 & 20 & 0\end{array}$

\section{Figure 1}


Kaplan-meier curves of long-term clinical outcome after the initial (A) and last (B) CA procedure between LINE-group and EGM-group.

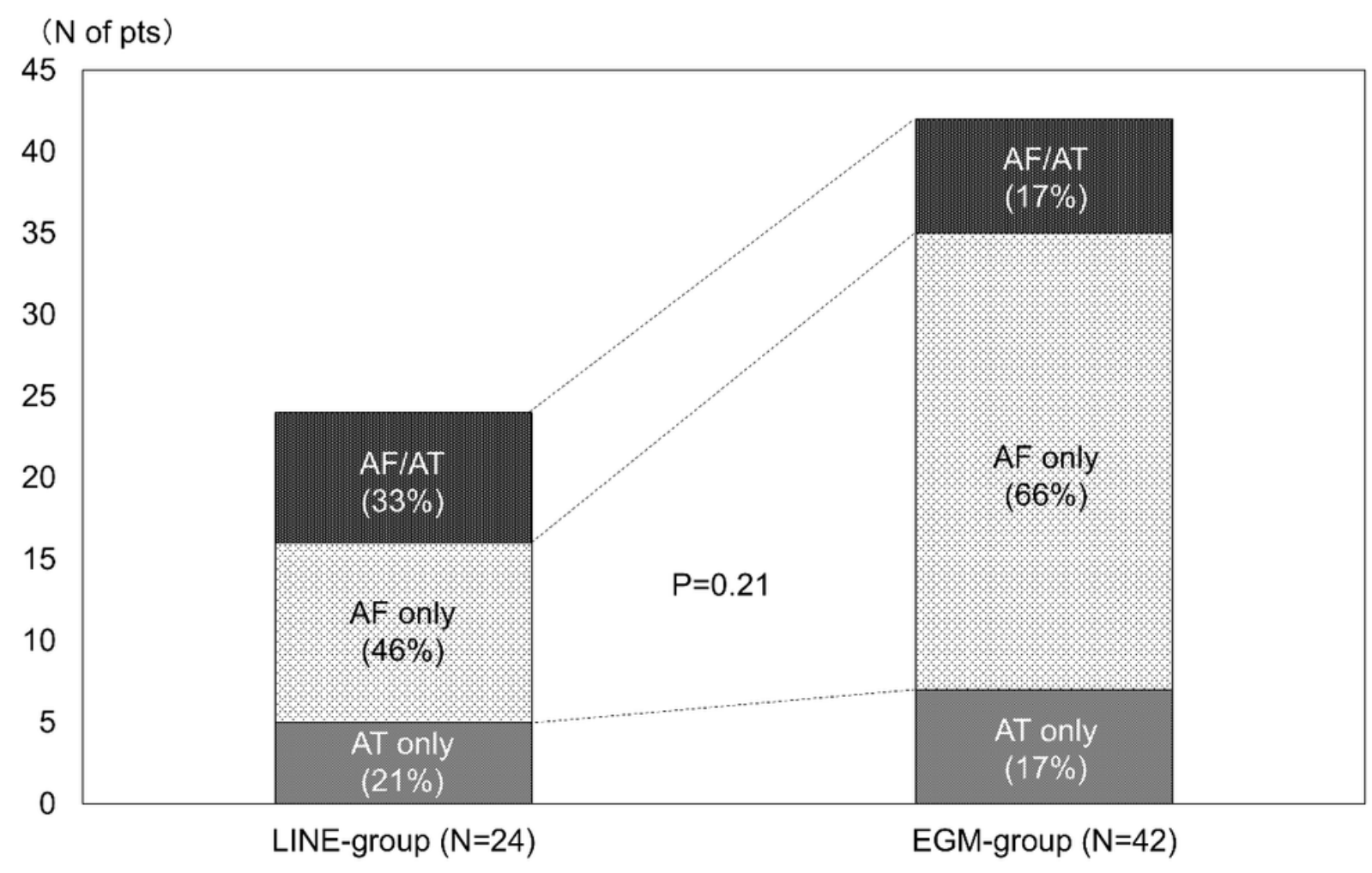

Figure 2

Recurrent forms after the initial CA between LINE-group and EGM-group. 